In addition to members of the Editorial Board, the individuals listed below provided constructive critical reviews of one or more manuscripts during the past year. Those who reviewed their first manuscript near the end of the year will be recognized in next year's acknowledgments. Appreciation for their valuable help to authors and the journal is expressed to the following reviewers:

Gloria Abad
Jorge Abad
George Abawi
Hamed Abbas
Adela Abelleira Argibay
Niwala Abeysekara
Nina Aboughanem-
Sabanadzovic
Maricelis Acevedo
Premila Achar
James Adaskaveg
Anthony Adesemoye
Tika Adhikari
Brenna Aegerter
Sonia Aghighi
Elizabeth Aitken
D. Soner Akgül
K. P. Akhtar
K. Akimitsu
Olufemi Akinsanmi
Maher Al Rwahnih
Khalil Al-Mughrabi
Abdullah Al-Sadi
Salam Al-thahabi
Olufemi Alabi
Hossein Alaei
Sandra Alaniz
Stephen Alderman
Ana Alfaro-Fernández
Acelino Alfenas
Mohammed Ali-Shtayeh
Akhtar Ali
Shaukat Ali
Caitilyn Allen
Rodrigo Almeida
Christie Almeyda
Anne Alvarez
Artur Alves
Masashi Amano
Achour Amiri
Mark Andersen
Didier Andrivon
Ravindran Aravind
Mohammad Arif
Valentine Aritua
Richard Baird
Yilmaz Balci
Arun Arya
Zahi Atallah
Herve Avenot
Yesim Aysan
Mohammad Babadorion-Montañestra
Ebrahiem Babiker
D. Backhouse
Charles Bacon

Peter Balint-Kurti

Sabine Banniza

Jeri Barak

Martin Barbetti

Charlie Barnes

Irene Barnes

R. W. Barreto

Daniel Bassimba

Kubilay Bastas

Anton Baudoin

Fulya Baysal-Gurel

Edward Beasley

Ivan Bedendo

Steven Beer

Franklin Behlau

Richard Bélanger

José Belasque Junior

Alessandra Belisario

Dirk Bellstedt

Celso Benedetti

Soledad Benitez-Ponce

Kemal Benlioglu

Rebecca Bennett

Mike Benson

Mónica Berbegal

Gary Bergstrom

Reinhard Berndt

Dana Berner

Assunta Bertaccini

David Beyer

John Bienapfl

Alan Biggs

Joseph Bigirimana

Regina Billones

Guillaume Bilodeau

Robert Blanchette

Bruce Bleakley

Charles Block

Vivian Blok

Cheryl Blomquist

Burt Bluhm

Clive Bock

William Bockus

Ana Bocsanczy

Pierluigi Bonello

Jorunn Børve

Steven Bost

Richard Bostock

Iraklis Boubourakas

Joseph Marie Bové

Robert Bowden

Kira Bowen

Claude Bragard

Mark Braithwaite

Uwe Braun

Nick Brazee

Rob Briddon

Ronald Brlansky

Kirk Broders

Marion Brodhagon
Philip Brown

Greg Browne

William Bruckart

Robert Brueggeman

James Buck

Alan Buddie

Carolee Bull

Russell Bulluck

Lindsey Burbank

Treena Burgess

Leon Burpee

Thomas Burr

Elizabeth Bush

Emmanuel Byamukama

Santa Olga Cacciola

Anthony Caesar

Guohong Cai

Rosa Caiazzo

Nora Caitlin

Marcos Camara

Mariano Cambra

Kim Camilli

Thierry Candresse

Didem Canik

P. Cannon

Lori Carris

Juliet Carroll

Lynn Carta

Frank Caruso

Pablo Castillo

Bertha Castro

Robert Cating

Nora Catlin

Raymond Cerkauskas

Chung Jan Chang

Amy Charkowski

Nikki Charlton

Gary Chastagner

Syama Chatterton

Michael Chatzidimopoulos

Ashok Chaudhury

Ravendra Chauhan

Shilpi Chawla

Fengping Chen

Jianchi Chen

Li-Fang Chen

Senyu Chen

ShuaiFei Chen

Weidong Chen

Xianming Chen

Yu Chen

Mingyuan Cheng

Xudong Cheng

Mohamad Chikh Ali

Periasamy Chitrampalam

Kishore Chittem

David Chitwood

Young-Joon Choi

Robin Choudhury

Wesley Chun
Kuang-Ren Chung

Maisa Ciampi-Guillardi

Aurelio Ciancio

Christian Cilas

Christopher Clark

Christopher Clarke

Keith Clay

Richard Cobb

Ron Cohen

Helvecio Coletta-Filho

Jack Comstock

Kassie Conner

Robbert Conner

Roger Cook

Daniel Cooley

Hans Cools

Len Coop

Warren Copes

Marco Cordero

James Correll

Sarah Costa

Laurent Costet

Teresa Coutinho

Brenda Coutts

Christina Cowger

Clayton Cox

Daniel Coyne

Julia Crane

Jodi Creasap Gee

Tom Creswell

Jo Anne Crouch

Pedro Crous

William Crow

Christian Cruz

Jaime Cubero

Marc Cubeta

Albert Culbreath

Washington da Silva

Abdelfattah Dababat

Leandro Dallagnol

John Damicone

Ulrike Damm

Louise-Marie Dandurand

Elizabeth Dann

Armelle Darrasse

Lawrence Datnoff

Margery Daughtrey

Robert Davidson

R. Michael Davis

Robert Davis

Antonieta De Cal

Filippo De Curtis

Leo De La Fuente

Maria de la Fuente

Erick De Wolf

Thomas Debener

Emerson Del Ponte

Luis del Rio Mendoza

Jill Demers

Sefer Demirbas 
Fikret Demirci

Michael Derie

Sibel Dervis

Cecile Desbiez

Megan Dewdney

Patrick Di Bello

Jose Dianese

Alfredo Diaz Lara

Gonzalo Díaz

Helene Dillard

Geoffrey Dixon

Gustave Djedatin

Aviv Dombrovsky

Leslie Domier

Weibo Dong

G. A. Dosio

Jim Downer

Mark Dragich

Martin Draper

Tyler Dreaden

Yongping Duan

I. M. Duarte

Nicholas Dufualt

Frank Dugan

Amara Dunn

Alan Dyer

Darin Eastburn

Daniel Egel

Al Eggenberger

Georgia Eizenga

Yigal Elad

Marianne Elliott

Monica Elliott

Wade H. Elmer

John Elphinstone

Lynn Epstein

Denna Errampalli

Paul Esker

Sydney Everhart

Kathryne Everts

Catherine Eyre

David Ezra

Roberto Faedda

Ahmad Fakhoury

Cesar Falconi

Bryce Falk

Mark Farman

Shirin Farzadfar

Xue Feng

J. Fernandes

Dolores Fernandez-Ortuno

Howard Ferris

Rosa Ferrucho

Elvira Fiallo-Olivé

Sabine Fillinger

Bruce Fitt

Jacqueline Fletcher

Francisco Flores

Xavier Foissac

Majid Foolad

Gregory Forbes

Helga Forster

Gerda Fourie

Adrian Fox

Stephen Fraedrich

Antonio Franceschini

Zachary Frederick

Stanley Freeman

Maira Freire

Jason French
Andrew Friskop

Ken Frost

William Fry

Marc Fuchs

Dennis Fulbright

Deanna Funnell-Harris

Gleiber Furtado

David Gadoury

Abraham Gamliel

Matteo Garbelotto

Maria Garcia-Pedrajas

Dominique Garcia

Denis Gaudet

Dhiraj Gautam

Klaus Geider

David Geiser

Thomas German

Mark Gibbs

Robin Giblin-Davis

Jessica Gigot

Robert Gilbertson

Paul Giordano

Ron Gitaitis

Dean Glawe

Cláudia Godoy

Katharina Gollner

Paolo Gonthier

Teresa Gonzalez-Jaen

Ana González

Paul Goodwin

Stephen Goodwin

Thomas Gordon

Bruce Gossen

S. Gowda

Jim Graham

David Gramaje

Giovanni Granata

Leah Granke

Stewart Gray

Nicola Greco

Michael Grisham

Ewald Groenewald

Rita Grosch

Niklaus Grünwald

Ganyu Gu

Walter Gubler

Neil Gudmestad

Lúcio Mauro Guimarães

Lodovica Gullino

Yongfeng Guo

Stephen Guy

Denita Hadziabdic

Geert Haesaert

Subhas Hajeri

Barbara Hall

Francois Halleen

Heather Hallen-Adams

Dennis Halterman

Jong Hyun Ham

Raymond Hammerschmidt

John Hammond

Rosemarie Hammond

John Hampton

Zafar Handoo

Mary Ann Hansen

Linda Hanson

Jianjun Hao

Tom Harrington

Nigel Harrison

Glen Hartman
John Hartung

Mohamed Hassan

Mary Hausbeck

Ryan Hayes

Hans Helder

Beatrice Henricot

Maria Henriquez

Ana Hernández-Lauzardo

Etienne Herrbach

Shaunta Hill

Martha Hincapie

Thien Ho

Bob Hoagland

Charles Hodges

Monica Hofte

Imre Holb

Gordon Holcomb

Ramon Holguin-Peña

Clayton Hollier

Gerald Holmes

Kathryn Homa

Bruce Horn

Brandon Horvath

Jeffrey Hoy

Tom Hsiang

Baishi $\mathrm{Hu}$

$\mathrm{Hao} \mathrm{Hu}$

Jiahuai Hu

Jianhuai $\mathrm{Hu}$

Xiufang $\mathrm{Hu}$

Li Huang

Don Huber

George Hudler

Julio Huerta-Espino

Gareth Hughes

Marc Hughes

Teresa Hughes

Scot Hulbert

Jodi Humann

Kevin Hyde

Takehumi Ikeda

Carlos Inacio

Patrik Inderbitzen

Russell Ingham

Debra Inglis

Laura Ingwell

Mário Inomoto

Renaud Ioos

Thomas Isakeit

Scott Isard

Yasushi Ishiguro

Takao Ito

Kelly Ivors

Allison Jack

William Jacobi

Barry Jacobsen

Courtney Jahn

Ramon Jaime-Garcia

Delano James

Wojciech Janisiewicz

Jacob Janse

Douglas Jardine

Yong Ho Jeon

Yulin Jia

Rafael Jiménez-Díaz

Maria Jimenez-Gasco

Elio Jimenez

Pedro Jimenez

Yue Jin

Young-Ki Jo
Charles Johnson

Dennis Johnson

Donn Johnson

Kameka Johnson

Steven Johnson

Jeff Jones

John Jones

Roger Jones

Katerina Jordan

Ramon Jordan

Hee-Young Jung

Wayne Jurick II

Makoto Kakishima

Sophia Kamenidou

Maria Kaponi

Rupam Kapoor

A. Karahan

George Karaoglanidis

Matthew Kasson

Pieter Kastelein

Nikolaos Katis

Sandi Keenan

Anthony Keinath

Lisa Keith

Heather Kelly

Gert Kema

Julia Kerrigan

Archana Khadgi

Youssef Khamis

Akhtar Khan

Mohamed Khan

Sebastian Kiewnick

Byoung Kyu Kim

Young Ho Kim

William Kirk

Levente Kiss

Nathan Kleczewski

Steven Klosterman

Steven Koike

Frederic Kolb

Andreas Kollar

James Kolmer

Ping Kong

Lise Korsten

James Kotcon

Liping Kou

Ondrej Koukol

György Kövics

Jan Kreuze

Branka Krstic

Gretchen Kuldau

P. Kumar

Madhurababu Kunta

James Kurle

Hadley Kutcher

Anders Kvarnheden

Panayota Kyriakopoulou

Chrostophe Lacomme

Ernesto Lahoz

David Lambert

James LaMondia

Sandra Lamprecht

Stephen Langrell

David Langston

M. Lapidot

Richard Latin

Jelena Latinovic

Bernardo Latorre

Daniel Lawrence

Ales Lebeda 
Jared LeBoldus

Jana Lee

Richard Lee

Su-Heon Lee

François Lefort

Anne Legrève

Carolina Leoni

C. Andre Levesque

Julien Levy

Morris Levy

Melanie Lewis Ivey

Baohua Li

Cheng-Wei Li

Fan Li

Hongye Li

Jinyun $\mathrm{Li}$

Ruhui Li

Shuxian Li

Ting Li

Wenbin Li

Yonghao Li

Orna Liarzi

Lia Liefting

Danielle Lightle

Magdalen Lindeberg

Steven Lindow

Kai-Shu Ling

Christopher Little

Jean Liu

Lianmeng Liu

Sanzhen Liu

Zhaohui Liu

Ioannis Livieratos

Williamn Livingston

M. Lobato

Benham Lockhart

Robert Long

Silvio Lopes

Carlos López-Herrera

Horacio Lopez-Nicora

Javier López-Robles

Silvia Lopez

Stefania Loreti

Frank Louws

Shien Lu

Gabriela Lucero

Jutta Ludwig-Mueller

Yong Luo

Maria Ma

Wenbo Ma

Steve Mackenzie

Mehrdad Madani

Laurence Madden

Robert Magarey

Clint Magill

Gaetano Magnano di San Lio

S. Mahadevakumar

Walter Mahaffee

Anne-Katrin Mahlein

Amer Mahmoud

George Mahuku

Dean Malvick

Sujan Mamidi

Michele Mansfield

Jose Marcelino

C. Marcone

Daniel Marcum

Hans Maree

Stephen Marek
Sasha Marine

Samuel Markell

James Marois

Robert Marra

Juliet Marshall

Frank Marthe

Frank Martin

Kathleen Martin

Robert Martin

S. Bruce Martin

Alfredo Martinez

Karunakaran

Maruthachalam

Sameer Masoud

Hayato Masuya

Michael Matheron

Febina Mathew

Louise May De Mio

Albert Mayfield

Gladys Mbofung

Robert Mc Intosh

Jenifer McBeath

Brent McCallum

Mary Ruth McDonald

Robert McGovern

Margaret McGrath

Eric McKenzie

Kevin McPhee

Neil McRoberts

Flavio Medeiros

Hilary Mehl

Lucky Mehra

Ulrich Melcher

José Melero-Vara

Alexandre Mello

Rachel Melnick-Lippart

Hassan Melouk

Michael Melzer

Fanhong Meng

Michelle Meng

Qingxiao Meng

Shawn Meng

Alemu Mengistu

Julien Mercier

James Mertely

Akos Mesterhazy

Monica Mezzalama

Themis Michailides

Sami Michereff

Manfred Mielke

Asimina Mila

Timothy Miles

Gerald Miller

Sally Miller

Zachariah Miller

Eugene Milus

Benjamin Mimee

Gaylord Mink

Toomo Misawa

Nathaniel Mitkowski

Margaret Mmbaga

Mojbata Mohammadi

Lucy Moleleki

Leire Molinero-Ruiz

Dimitre Mollov

Ian Moncrief

Mauricio Montero-Astúa

Concepció Moragrega

Lisela Moreira

Erique Moriones
Dimitrios Moshou

Manuel Mota

Lacey Mount

Andrea Moura

Enersto Moya-Elizondo

Weston Msikita

Hans-Peter Muehlbach

Daren Mueller

R. Mumford

Francoise Munaut

Christopher Mundt

Gary Munkvold

Joseph Munyaneza

John Murphy

Rita Musetti

Jim Myers

Roxana Myers

Russell Nagata

Peter Nagy

Masami Nakajima

Tomohide Natsuaki

Cristian Nava-Diaz

Jesús Navas-Castillo

Shrishail Navi

Monica Navia-Urrutia

Kumarse Nazari

Stephen Neate

Berlin Nelson

Eric Nelson

Terry Niblack

Paul Nicholson

P. Nicot

Mizuho Nita

James Noe

Joseph Noling

Phillip Nolte

Jay Norelli

David Norman

Phillip Northover

Glauber Nunes

Joe Nunez

Richard Nyankanga

Kerry O'Donnell

Cynthia Ocamb

Francisco Ochoa-Corona

Peter Ojiambo

Patricia Okubara

Mitsuru Okuda

Gilberto Olaya

Helena Oliveira

Chrystel Olivier

Mary Olsen

Brian Olson

Kevin Ong

Angela Orshinsky

Juan Osorno

Manuel Ospina-Giraldo

Nancy Osterbauer

Bonnie Ownley

Aaron Palmateer

Cristi Palmer

Lluís Palou

Xuebiao Pan

Ireneo Pangga

Dilip Panthee

Hanu Pappu

Mathews Paret

Päivi Parikka

Dong Suk Park

Giuseppe Parrella
Julie Pasche

Ana Pastrana

Jaimin Patel

Andrea Patocchi

Timothy Paulitz

Andrea Payne

Tamieka Pearce

Mike Pearson

John Pecchia

Francesca Peduto Hand

Tobin Peever

Yan Pei

Juan Peña-Cabriales

Analía Perelló

Alejandro Perez-Garcia

Ana Pérez-Sierra

B. Perez-Vich

Juliana Irina Perminow

Keith Perry

Paula Persson

Ramasamy Perumal

Zvezdana Pesic

Kari Peter

Sarah Pethybridge

William Pfender

Ludwig Pfenning

Minna Pirhonen

Tuula Piri

Antoon Ploeg

James Polashock

Anissa Poleatewich

Giancarlo Polizzi

Jane Polston

Grant Poole

Lyndon Porter

Bindu Poudel

Edson Pozza

Zacharias Pretorius

Jaroslava Pribylova

Trey Price

Claudia Probst

Simone Prospero

Dov Prusky

Barry Pryor

Jay Pscheidt

Michael Pumphrey

Zamir Punja

Guoliang Qian

Feng Qu

Fabio Quaglino

Diego Fernando QuitoAvila

Sebastjan Radisek

Cayo Ramos

Sephra Rampersad

G. Rao

Rosa Raposo

John Rascoe

Arash Rashed

Claudio Ratti

Abbasali Ravanlou

Min Rayamajhi

Naidu Rayapati

Peg Redinbaugh

Cecilia Rego

Silvia Restrepo

Marie Emma Rey

Luca Riccioni

Gabriela Ritokova

David Rizzo 
Joseph Roberts

\section{P. Roberts}

Steven Roberts

Fabricio Rodrigues

Jeffery Rollins

Gianfranco Romanazzi

Megan Romberg

Suzanne Rooney-Latham

David Rosenberger

Vittorio Rossi

Erin Rosskopf

Amy Rossman

Dorith Rotenberg

Craig Rothrock

Phillipe Rott

Tatiana Roubtsova

Douglas Rouse

Matthew Rouse

Thierry Rouxel

Avijit Roy

Daniel Royse

Gail Ruhl

John Rupe

Charlie Rush

Sead Sabanadzovic

Ivan Sache

Ivana Šafránková

Aniruddha Saha

Maria Sainz

Seiya Saito

Pasquale Saldarelli

Deborah Samac

Abdul Samad

Pedro Sánchez-Peña

Paloma Sanchez-Torres

Ethel Sanchez

Randy Sanderlin

H. Sanfacon

Yoshitaka Sano

Soum Sanogo

Luisa Santamaria

Maria Saponari

Arvind Saroj

Panagiotis Sarris

Toyozo Sato

Mamoru Satou

Kyryll Savchenko

María Scandiani

Sara Schaarschmidt

Kay Scheets

Leonardo Schena

Harald Scherm

William Schillinger

David Schmale

Guido Schnabel

Raymond Schneider

William Schneider

Alexandra Schoeny

Markus Scholler

Kurtis Schroeder

Howard Schwartz

Jason Scott

Simon Scott

Gary Secor

Kenneth Seebold Jr.

Marion Seier

Venkatesan Sengoda

Cigdem Serce

Luz Serrato-Diaz

Denis Shah
Muhammad Shahid

Simon Shamoun

Gunjan Sharma

Mamta Sharma

David Shaw

Kenneth Shenge

Barbara Shew

David Shew

Ainong Shi

Ryoji Shinya

Nina Shishkoff

Dylan Short

Dani Shtienberg

Yasmeen Siddiqui

Edward Sikora

Devendra Singh

Jitender Singh

Mathuresh Singh

Raghuwinder Singh

Ganga Devi Sinniah

Brent Sipes

Felipe Siverio

Andrea Skantar

Bernard Slippers

Christine Smart

Joseph L. Smilanick

Richard Smiley

Barbara Smith

Denise Smith

Tom Smith

Baruch Sneh

Richard Sniezko

Dartanha Soares

Mark Sosnowski

André Souza

Adam Sparks

Christoffel Spies

Otmar Spring

Terry Spurlock

Shikha Srivastava

James Stack

Marciel Stadnik

Gerd Stammler

Michael Stanghellini

Glen Stanosz

Karl Steddom

Sebastian Stenglein

Katherine Stevenson

Jane Stewart

Lucy Stewart

Silvina Stewart

G. Stirling

Jeffrey Stone

Becke Strehlow

Stephen Strelkov

Gunn-Mari Strømeng

Rona Sturrock

Sergei Subbotin

Mysore Sudarshana

Haruhisa Suga

David Sugar

Serenella Sukno

Grace Sumampong

Brett Summerell

George Sundin

Turner Sutton

Suwandi Suwandi

Wijnand Swart

Bryan Swingle

Kylie Swisher
Les Szabo

Stephanie Szostek

Girma Tabor

Susumu Takamatsu

Yuichi Takikawa

Miguel Talavera

Nobuya Tashiro

Satyanarayana Tatineni

Jeanette Taylor

Paul Taylor

Robert Taylor

S. Taylor

Eric Tedford

Albert Tenuta

Adriana Teramoto

Tarekegn Terefe

Antonio Testa

Yared Tewoldemedhin

Thanuja Thekke Veetil

Judy Thies

Tarlochan Thind

Marco Thines

Carla Thomas

Thomas Thomidis

Jeremy Thompson

John Thompson

Bin Tian

Tongyan Tian

Xiuling Tian

L. W. "Pete" Timmer

Patricia Timper

Ned Tisserat

Sotiris Tjamos

Steven Tjosvold

Napoleon Tjou-Tam-Sin

Sue Tolin

Laura Tomassoli

Jennifer Tomlinson

Paul Tooley

Chris Toomajian

Ivo Tosevski

Juan Manuel TovarPedraza

Lane Tredway

Larry Trevathan

Robert Trigiano

Lindsay Triplett

Pankaj Trivedi

Florent Trouillas

Mina Tsagris

Jwu-Guh Tsay

Leah Tsror

Kayimbi Tubajika

Elena Turco

William Turechek

Massimo Turina

T. Tworkoski

Lydia Tymon

Janice Uchida

Dhanushka Udayanga

Sai Sree Uppala

Inmaculada Vaca

Demetrios Vakalounakis

Alberto Valencia-Botin

Jari Valkonen

Gary Vallad

Rodrigo Valverde

Ariena Van Bruggen

Esther van den Berg

T. van der Lee
Rene Van der Vlugt

Jacquie van der Waals

Jan Van der Wolf

David Van Sanford

George Vandemark

Joel Vanneste

Christina Varveri

Nikon Vassilakos

Byron Vega

Aline Velho

Vittorio Venturi

Jeanmarie Verchot

Valérie Verdier

Joseph Verreet

Antonio Vicent

Joana Vicente

Georgios Vidalakis

Dan Villamor

Dan Edward Villamor

Sara Villani

Boris Vinatzer

Paul Vincelli

Detlev Vogler

Andreas Voloudakis

Susanne von Bargen

Anne-Sophie Walker

David Walker

Greg Walker

Nathan Walker

Ann-Charlotte

Wallenhammar

Christopher Wallis

Yeshi Wameshi

Anmin Wan

Guo-Liang Wang

Jinbo Wang

Meinan Wang

Xuefeng Wang

Leslie Wanner

Astri Wayadande

Mark Weaver

Kimberly Webb

Stephen Wegulo

John Weiland

Bevan Weir

Ruth Welliver

Aimin Wen

Sabine Werres

Todd West

Andreas Westphal

Alan Westra

Henry Wetzel III

Phillip Wharton

Terry Wheeler

Vance Whitaker

Frank White

Melanie Whitelaw-Weckert

Steven Whitham

Robert Wick

Valerie Williamson

Laetitia Willocquet

Mark Windham

William Wintermantel

Johanna Witzell

Silvia M. Wolcan

Charles Woloshuk

Alan Wood

James Woodhall

Jason Woodward

Eduardo Wright 
Martin Wubben

Christian Wyenandt

Ye Xia

Chang-Lin Xiao

Zhongguo Xiong

Xiangming $\mathrm{Xu}$

Chunsheng Xue

Mohammad Yaghmour

Lani Yakabe
Guiping Yan

X. B. Yang

Xiao Yang

Weimin Ye

Xujun Ye

Shyi-Dong Yeh

Keith Yoder

Carolyn Young

Qing Yu
Laércio Zambolim

Kurt Zeller

Quan Zeng

Marcelo Zerillo

Jianhua Zhang

Ning Zhang

Shouan Zhang

Shujian Zhang

Yunzeng Zhang
Bingyu Zhao

Boguang Zhao

Shaobin Zhong

Erxun Zhou

Jing Zhou

Mingguo Zhou

Nina Zidack

Thomas Zitter

Shimin Zuo 